\title{
Parallel Adaptive Simplical Re-Meshing for Deforming Domain CFD Computations
}

\author{
Sandeep Menon ${ }^{\mathrm{a}}$, Kyle G. Mooney ${ }^{\mathrm{a}}$, K.G. Stapf ${ }^{\mathrm{b}}$, David P.Schmidt ${ }^{1 \mathrm{a}}$ \\ ${ }^{a}$ Department of Mechanical and Industrial Engineering, University of Massachusetts \\ Amherst, 160 Governors Dr., Amherst MA 01003, USA \\ ${ }^{b}$ Ingenieurbüro TWB, Fulda Germany
}

\begin{abstract}
Deforming domains occur in many fields of computational fluid dynamics (CFD), such as interface tracking, simulation of pumps and engines, and fluid/structure interaction. The deformation of the domain presents a challenge to the integrity of the computational mesh; substantial motion of the domain boundaries requires vertex motion and changes in mesh connectivity. For cases of simple boundary motion or structured meshes, predetermined changes to the mesh structure can be sufficient. However, without a priori knowledge of how the domain will change, a more robust solution is required. The present work offers a parallelized solution for simplical meshes that is well-suited to extremely complex geometry. The mesh continuously evolves without user intervention or the use of target meshes. Varying length scales imposed by evolving boundary curvatures and narrow gaps are resolved with a fast length-scale algorithm. The set of algorithms are incorporated into an object-oriented code structure that permits broad application to a range of CFD problems. The robustness and versatility of the algorithm is demonstrated in several examples, representing motion of internal and external boundaries, where the boundary motion may or may not be known a priori.
\end{abstract}

Keywords: CFD, adaptive remeshing, parallel computing, conservative remapping, mesh smoothing, local mesh optimization

\section{Introduction}

Many classes of fluid-flows, such as fluid-structure interaction, reciprocating pumps and engines, and free-surface flows, involve deforming fluid domains. Computational fluid dynamics (CFD) simulations in these deforming domains present several challenges. The foremost challenge is how to maintain adequate mesh quality while the shape of the domain, and hence interior cells, undergoes large changes. Even a single poor-quality cell is sufficient to halt a simulation, so

\footnotetext{
${ }^{1}$ Corresponding Author: schmidt@ecs.umass.edu
}

Preprint submitted to Journal of Computational Physics

May 28, 2015

(C) 2015. This manuscript version is made available under the Elsevier user license http://www.elsevier.com/open-access/userlicense/1.0/ 
robust techniques are required that allow the mesh to adapt while maintaining good cell quality.

In general, transformations or mesh motion are not sufficient; adaptive remeshing (or re-gridding) an existing mesh will eventually be required. In the framework of simulations with boundary deformation such re-meshing methods are often necessary if cells become excessively distorted and mesh smoothing cannot mitigate the issue. One approach is to re-mesh the domain entirely using an appropriate mesh-generation algorithm. There are two major drawbacks to this approach: First, mesh-generation can be a particularly time-consuming, difficult process. Second, re-meshing requires the interpolation of flow variables to the new mesh, which can frequently induce errors and instabilities to the solution. By re-meshing the entire domain, these errors can often be difficult to contain, and may magnify as the simulation proceeds over time. For computational studies involving rigid boundary motion or complex boundary fitted meshes, the overset grid approach can be an attractive choice $[1,2,3]$. In the case of a deforming boundary, however, an overset mesh would still suffer from the same distortion effects.

An alternative approach is local re-meshing, which works well in minimizing interpolation errors and, given the right algorithms, can be quite effective in both finite volume [4] and finite element [5] applications. The local re-meshing includes both mesh motion and connectivity changes [6]. Although some interpolation error does occur, this error will be limited to regions where changes in mesh connectivity are necessary. In contrast, global re-meshing incurs error throughout the domain.

The topic of mesh reconnection can also be extended to include refinement and derefinement of cells $[7,8,9,10]$. Physical phenomena can often develop over varying length scales, with near-singular solutions and large gradients in very localized regions, and in most cases, the only solution is to resolve these variations using an increased number of cells in the area. The option of uniformly refining the entire mesh is usually rejected as the large increase in computational effort (particularly in three dimensions) cannot be justified, and areas away from the problematic region may require far less local mesh resolution. Local refinement allows an increase in mesh density around areas that need it most, thus providing improved solution accuracy at a more acceptable computational cost. For examples of serial adaptive meshing in the finite-element community see [5], [11] and [12]. More recently, the work of Clausen et al. [13] offers an example of highly complex boundary motion in a serial free-surface computation. The application to free-surface flows is especially compelling when high-fidelity treatment of the interface is required, such as in Dieter-Kissling et al. [14].

For fixed boundary positions, oct-tree based methods can employ refinement and coarsening and simultaneously achieve excellent parallel speedup on thousands of processors [8]. This parallel speed-up enables highly-refined computations bridging variable length scales [15]. The limitation is that large and complex boundary deformation is not included in these simulations. If the mesh is deformed to accommodate large boundary deformation, interior cells would become twisted and degenerate. 


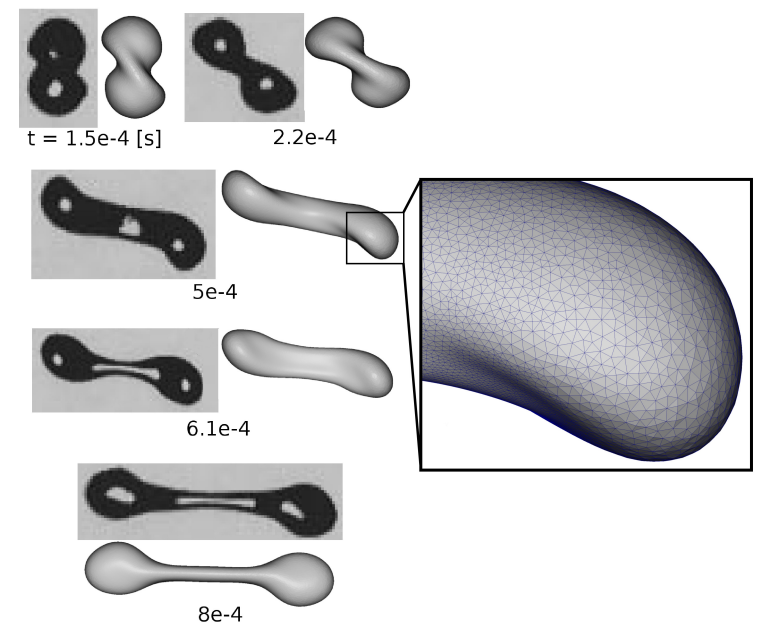

Figure 1: A validation showing the adaptive tetrahedral mesh paradigm applied to simulations of droplet collisions. The experimental images are from Qian and Law [21] and the computations are from Mooney et al. [17]

In contrast, the present work is more intended to handle parallelism where boundary deformation is complex or not a priori known. The approach is based on simplical meshes; all cells and faces are simplexes. Validation of serial computations based on our fully-adaptive simplicial mesh approach can be found in numerous works: Dai and Schmidt [4], Quan et al. [16], Mooney et al. [17], Mooney and Schmidt [18], Dieter-Kissling et al. [14], Dieter-Kissling et al. [19], and Batta et al. [20], among others. A validation example is shown in Fig. 1. The next, natural step, after extensive serial use, is to extend this paradigm to larger-scale computation.

The present work demonstrates a parallelized method of adapting the mesh that can handle large boundary deformation, variable resolution requirements, and conservative transfer of cell-centered fields to the new mesh. The novel contribution of this work is the parallelization of this adaptation method using a domain decomposition approach. These combined capabilities allow this adaptive mesh method to handle the a challenging class of problems where numerous boundaries deform and move in ways that may not be known prior to the actual computation.

The requisite changes in mesh topology complicate the parallelization paradigm, which relies on decomposing the larger domain into numerous sub-domains. Extensive mesh changes on inter-processor boundaries are an extreme challenge, since these changes can involve data structures residing on several processors [8]. The most similar work to-date, by Cavallo et al. [22], avoided this issue through large-scale transfer of cells from one processor to another. The present work takes a more local approach in order to reduce communication costs between processors. 
The means of accomplishing this local approach are discussed below and followed with a variety of demonstration calculations. First, the basic elements of mesh adaptation are described. Next, the methodology explains how these elements are implemented in parallel. Finally, the algorithm is demonstrated and tested in a finite-volume framework. The results include speedup tests and validation of the computations.

\section{Mesh adaptation strategies}

The crux of the moving mesh method is to maintain mesh quality while the boundaries of the domain impose deformation. The mesh can respond in two ways to required boundary constraints, as exemplified by the work of Klinger and Shewchuk [6]. The mesh can move in a manner that maximizes mesh quality, which requires a calculation of vertex motion, as described in Section 2.1. Additional examples from the literature include Erzincanli and Sahin [23] and Bos [24]. The examples of Brusiani et al. [25] and Lucchini et al. [26] are particularly relevant since, like the present work, they were tested on internalcombustion engine motion.

The other method for improving the mesh requires changes to mesh connectivity, which may be driven by several considerations. In certain situations, neighboring vertices may move prohibitively far apart or may become nearly co-located. In these cases, the mesh connectivity must change to allow the simulation to continue, as described in Section 2.2.

Further, the insertion or deletion of mesh elements, which are forms of mesh connectivity changes, can be used to refine or coarsen the mesh. The algorithms for these operations are given in Section 2.3. The length scale used to judge the mesh resolution, and decide whether refinement or coarsening is warranted, is explained in Section 2.4 .

\subsection{Mesh Smoothing}

Mesh smoothing, sometimes referred to in literature as mesh motion, or mesh re-zoning, is the process of mesh improvement through vertex motion. An effective smoothing technique continuously maintains the quality of the mesh during the course of the simulation, and can often delay the requirement for local reconnection, which in turn minimizes interpolation errors. Pure mesh motion (i.e., without any re-meshing) can easily be incorporated into conservation laws by using relative fluxes, as specified by the Reynolds Transport Theorem.

To maintain the quality of cells away from mesh boundaries towards the interior, this work uses the Mesquite Mesh Improvement library from Sandia National Labs [27] for three dimensional meshes. The library is independent by design and can be linked via functional interfaces, thereby making it versatile and highly efficient.

Mesquite cannot, however, optimize vertex positions on complicated boundaries like the situations involved in this work, and assumes that these points on the input mesh are fixed. Thus, surface smoothing is first performed prior to the optimization of interior points. 
Surface smoothing is achieved by using a variant of the spring-analogy based Laplacian approach (Blom [28], Huang and Russell [29]) - a simple algorithm that is quick, both in implementation and run-time efficiency. In its basic form, this method moves every vertex in the mesh to the average position of its nearest neighbors, and is governed by the minimizing the following function:

$$
\left(\mathbf{I}-\mathbf{n n}^{T}\right) \sum_{j} k_{i j}\left(\mathbf{x}_{i j}-\mathbf{x}_{i}\right)=0
$$

where, $\mathbf{x}_{i j}$ denotes the position of every neighboring vertex immediately connected to vertex $\mathbf{x}_{i}, \mathbf{n}$ is the unit point-normal vector at the surface, and $k_{i j}$ represents a spring constant. This is analogous to the case where edges connecting two neighboring vertices are regarded as springs in tension, and are allowed to relax to their equilibrium state. This is a well-posed energy minimization problem that can be solved effectively using the Conjugate Gradient method.

One potential difficulty of surface smoothing, however, is that the surface mesh motion has implications for interior mesh quality. Since the motion of surface points is governed by the surface springs, interior cell quality could be compromised. If mesh motion would cause interior cells to invert, underrelaxation was employed to restrain the surface point motion.

\subsection{Adaptive Reconnection for Improved Mesh Quality}

The quality of a simplical mesh can be locally improved by an operation known as edge-swapping (or edge-flipping), which is applicable in both twoand three-dimensions. In two-dimensions, the condition for edge-swapping is defined by the Delaunay criterion, which specifies that no mesh points are to be contained in the circumcircle of any cell of the mesh. Unfortunately, the Delaunay criterion is not sufficient in three dimensions, though recent work shows the potential for the incorporation of additional constraints [30]. Consequently, the present work relies upon an optimization-based method for local connectivity improvement $[31,6]$.

In three-dimensions, edge-swapping consists of three elementary operations: 2-3 and 3-2 swaps as shown in Fig. 2 and the 2-2 swap as shown in Fig. 3. Topologically, this is considerably more complicated when compared to the $2 \mathrm{D}$ case. In a tetrahedral mesh, an edge can be connected to an arbitrary number of cells and so it becomes unclear how an edge-swapping operation is to be implemented in a general manner. One of the first proposed solutions to this problem was defined by Briere de L'isle and George [32], called the edge-removal operation, which removes a single edge from the mesh (and therefore, all the tetrahedra connected to it), and replaces the local void with a new configuration of tetrahedra with an improved mesh quality.

The algorithm used in this work is an optimization-based approach which produces the locally optimal edge configuration in polynomial time $[31,6$, 4]. The algorithm uses a dynamic programming approach which was originally introduced by Klincsek [33], and does not impose an upper bound on the number of cells surrounding an edge. The edge removal process consists of a sequence 


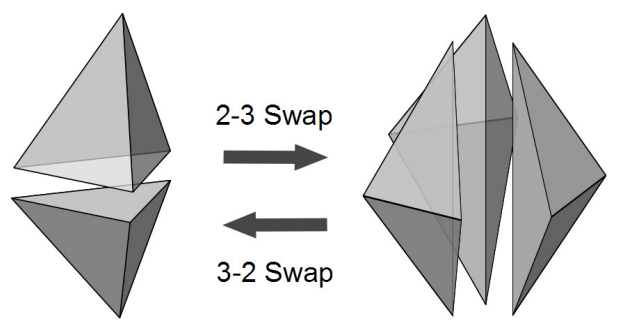

Figure 2: Complementary 2-3 and 3-2 swapping operations in 3D

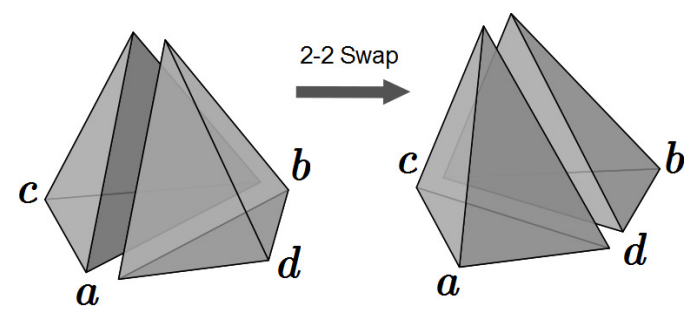

Figure 3: Surface 2-2 swapping operation in 3D, where vertices a-d lie on a mesh surface

of $(m-3)$ 2-3 swaps followed by a final 3-2 swap, where $m$ is the number of cells surrounding a shared edge. For more information readers may refer to the work by Freitag and Ollivier-Gooch [31], Klinger and Shewchuk [6], and Dai [4].

\subsection{Adaptive Reconnection for Length Scale Resolution}

Mesh refinement can be performed in a variety of ways. The most common approach, also taken in this work, is the h-refinement method, which involves the addition of points at regions which require higher mesh density to resolve high solution gradients. Derefinement (or the removal of points) is also possible in regions where the solution error is low. Examples of h-refinement can be found in work by Jasak [34], Muzaferija [35], and Coelho et al [36], among others.

The two important operations required for h-refinement in simplical 2D and 3D meshes, bisection and contraction, are shown in Fig. 4 and Fig. 5. Face trisection, an additional refinement feature specific to $3 \mathrm{D}$ tetrahedral meshes, is shown in Fig. 6. In this case, h-refinement calls for coarsening and refinement are dictated by a target length scale for each edge. Should an edge's length deviate from its target, it will be flagged for h-refinement. In this work, the target length scale is set at the domain boundaries and propagated into the interior mesh through a face-cell wave, however, many other successful approaches to calculating target length scale fields have been presented $[11,12,10]$. The method by which the length scale is estimated does not change the underlying topological algorithms. 

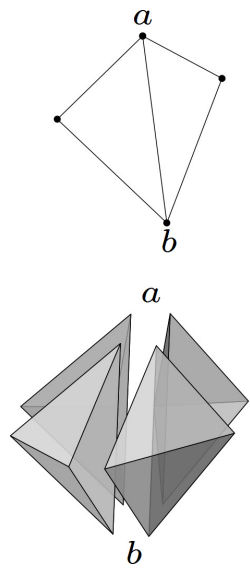

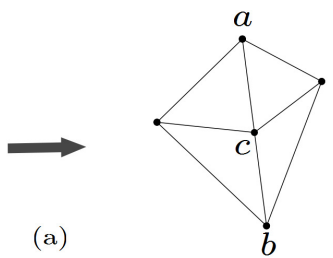

(b)

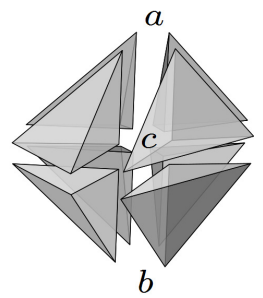

Figure 4: Edge bisection in (a) 2D; and (b) 3D
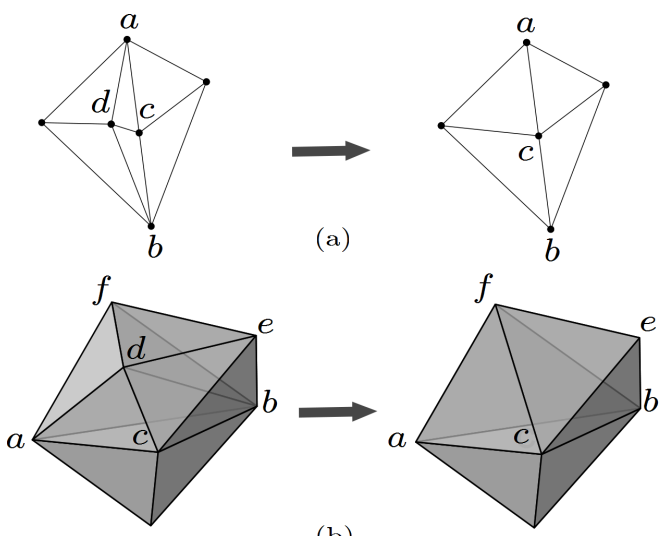

(b)

Figure 5: Edge contraction in (a) 2D; and (b) 3D
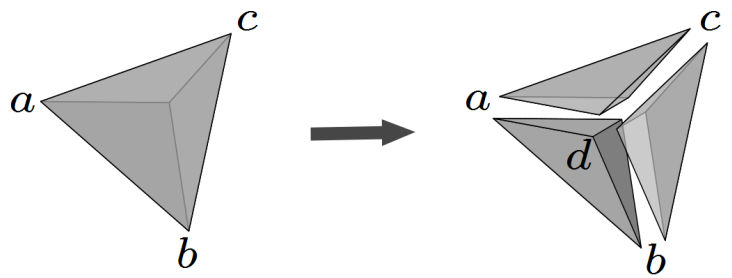

Figure 6: Face trisection in 3D 


\subsection{Estimation of Local Length Scale}

Prior to an adaptive mesh refinement procedure, it is important to define a criterion for which points are to be added to (or removed from) the mesh. While there are several choices (such as solution error, for example), the criterion used in this work is based on an estimate of the local length scale field $\mathbf{L}(\mathbf{x})$, which is a scalar value defined for every cell in the mesh.

Often, the length scale is determined locally from gradients or estimation of numerical errors. The present work focuses on boundary-driven flows, which is consistent with the emphasis on moving, deforming boundaries. For such flows, the length scale should posses the following two properties:

- The value of $\mathbf{L}(\mathbf{x})$ at $\mathbf{x}=\partial \Omega$ (where $\partial \Omega$ is the boundary of the solution domain $\Omega$, such as a fluid interface), must be sufficiently small so that the surface curvature is captured with sufficient accuracy. The length scale must not be too small, so as to increase numerical stiffness induced by a Courant number restriction.

- Also, $\mathbf{L}(\mathbf{x})$ for $\mathbf{x} \in \Omega$ must gradually increase as $\mathbf{x}$ tends away from $\partial \Omega$ towards the interior, so that computational effort for the solution is reduced.

Dai [37] used a spatially varying vector indicator function to achieve an estimate for $\mathbf{L}(\mathbf{x})$. This approach requires the solution to a discrete vector Laplace equation at each time-step (albeit with a relaxed tolerance for the iterative solver), which is comparable to the cost of the pressure Poisson equation.

The algorithm devised in this work uses a greedy approach in conjunction with mesh connectivity information, and requires a length scale value to be specified for boundary faces of the domain [38]. The fixed-value length scale is not required to be specified for all boundary faces, but must be specified for at least one of them. A growth-factor may be provided by the user which specifies how quickly the cell-size is expected to grow toward the interior of the mesh.

\subsection{Estimation of Boundary Length Scale}

The algorithm specified in Section 2.4 requires a fixed-value length scale to be defined at least one boundary face in the domain, based on which length scales at the interior of the mesh are established. This length scale can be specified in various ways, and the following criteria have been used in this work:

Constant surface length scale, $\left(\mathbf{b L}_{f}\right)$ : The choice of a constant length scale allows a fixed edge length to be maintained at the surface during the course of the simulation. This approach will typically fail to refine the surface adequately at interesting regions (involving high curvature, for example), and is almost always combined with other boundary length scale specifications. 
Length scale dictated by local curvature, $\left(\mathbf{b L}_{\kappa}\right)$ : Local curvature at a specified surface edge is roughly estimated by comparing the normals of the two faces connected to it. If the projection of one normal on the other falls below a specified fraction, the edge is marked for refinement.

Length scale dictated by proximity to a surface $\mathcal{S},\left(\mathbf{b L}_{p}\right)$ : In certain situations like droplet coalescence, where a narrow space lies between two surfaces, it is often desirable to refine the approaching surfaces so that the transition for interface topology changes is relatively smooth. This would require the length scale at these sections to be defined by the proximity to the approaching surface. Clearly, checking for the distance to every other edge on the interface is impractical and therefore, a reduced search algorithm is implemented using a 3D hash algorithm, where the space around a surface is divided into bins for faster computation. Proximity of the specified surface edge to $(\mathcal{S})$ is then calculated by the following steps:

1. The edge-normal $\left(\mathbf{n}_{e}\right)$ is calculated for each edge by adding the normals of two boundary faces adjacent to it.

2. With a step-size of the edge length, take multiple steps in the edgenormal direction and perform a spatial hash of each step to obtain the corresponding bin. This bin is added to the list of bins to be checked.

3. Now loop through all bins and calculate the distance to faces in each bin, and select the minimum distance $\left(d_{\min }\right)$ to opposing face candidates. Opposing face candidates (with a face normal, $\mathbf{n}_{o f}$ ), is defined such that $\mathbf{n}_{\text {of }} \cdot \mathbf{n}_{e} \approx-1.0$.

4. The length scale at the edge location is now defined as $\xi d_{m i n}$, where $\xi$ is a multiplicative constant (chosen to be 0.2 in this work).

Length scale dictated by time-step restrictions, $\left(\mathbf{b L}_{t}\right)$ : To avoid problems associated with excessive numerical stiffness and stability, the overall minimum mesh length scale may have to be limited to a certain value, such as one dictated by a Courant number restriction.

\section{Parallel Mesh Adaptation}

Having defined the elementary operations required to maintain mesh quality and resolution, the remainder of the methodology is devoted to describing how to implement these ideas in a distributed computational framework. The distributed approach to parallel mesh adaptation considered in this work is achieved using the Message Passing Interface (MPI) paradigm [39]. This involves the partitioning of the computational mesh into several sub-domains which represent a portion of problem being solved. 


\subsection{Domain Decomposition and Halo meshes}

The first step in the distributed memory approach is to decompose the solution domain into several parts, such that each sub-domain is allocated to a processor. Several popular examples of work in this area include the METIS library by Karypis and Kumar [40], the Zoltan library by Devine et al. [41], and the Scotch partitioning library by Pellegrini and Roman [42]. Although the partitioning process is often considered to be a pre-processing step for most static parallel runs, simulations that dynamically change the mesh structure during run-time must also occasionally re-partition the domain due to the gradual imbalance in sub-domain cell counts over time.

Prior to any mesh adaption procedure, each sub-domain must have a minimal description of the mesh structure connected to processor-boundary points on neighboring sub-domains, defined as a halo mesh. Note that a 'neighbor' sub-domain in this context can share points, edges or faces. In addition to basic connectivity structures, each halo mesh must also carry a description of its boundary patches, since this information is often necessary during the adaptation procedure. Quite often, decomposed domains contain points that are simultaneously shared by several processors at the same time, known as global points. These points must first be identified in order to assist in the halo mesh construction process. In this work, these points are identified by an efficient algorithm developed by Jasak [43] that uses only nearest-neighbor information. Halo meshes are then defined for each processor interface, consisting of any tetrahedra that shares a point, edge, or face with a processor boundary.

Once halo meshes have been constructed, each sub-domain must send and receive them to neighboring sub-domains using MPI. This transfer can be done asynchronously, so each sub-domain is free to do other useful work while transfers occur in the background. After all transfers have completed, each subdomain must prepare entity maps that relate points, edges and faces on processor boundaries with equivalent entities on the received halo meshes, and vice-versa. Thus, for example, a point map $\mathscr{X}_{p}^{j}: \mathscr{N} \rightarrow \mathscr{N}^{j}$ takes a point from the sub-domain point-set $(\mathscr{N})$ to its equivalent point on the halo mesh point-set $\left(\mathscr{N}^{j}\right)$ from processor $j$, while a reverse point map $\mathscr{R}_{p}^{j}: \mathscr{N}^{j} \rightarrow \mathscr{N}$ does the opposite. Similar maps are constructed for edges $\left(\mathscr{X}_{e}^{j}, \mathscr{R}_{e}^{j}\right)$ and faces $\left(\mathscr{X}_{f}^{j}, \mathscr{R}_{f}^{j}\right)$. Naturally, each entity map is defined for only a subset of all entities on each sub-domain.

\subsection{Parallel Mesh Smoothing}

This section will discuss the various details of the mesh smoothing algorithms presented in Section 2.1, when extended to a distributed-memory paradigm. The topic of parallel mesh smoothing has been investigated by several researchers in the past. Freitag et al. [44] extended their local-optimization algorithm to a Parallel Random Access Machine (PRAM) computational model, which allows sub-domains to access other sub-domains in a shared-memory paradigm. Tsai et al. [45] used a transfinite interpolation (TFI) technique to smooth multi-block meshes in parallel, with a minimal amount of inter-processor communication. 
Jiao et al. [46] extended a feature-preserving surface-mesh smoother to a distributed memory model, and observed near-linear scaling for up to 128 processors using a Myrinet interconnect. Guoy et al. [47] used a halo mesh approach to extend the Mesquite Mesh Improvement Library to perform smoothing in parallel.

Like the serial treatment, the parallel smoothing process begins with the surface. In contrast to the work by Jiao et al. [46], where surface-mesh vertices are moved in a localized manner, the approach taken in this work is to perform a global repositioning of surface vertices by extending the spring-analogy Laplacian smoothing method described in Section 2.1 to a distributed-memory paradigm. The relation governing the position of surface vertices is given by Eq. 1. When the point-normals are treated explicitly, the relation in Eq. 1 yields a symmetric positive-definite matrix of coefficients which can be solved efficiently using the Conjugate Gradient method [48].

The approach taken by Gouy et al. [47] is used in this work for the parallel smoothing of interior vertices using Mesquite. The preparation of halo meshes for parallel smoothing is identical to the description given in Section 3.1, which is illustrated in Fig. 7. As shown in the figure, all points on the boundary of the sub-domain are held fixed (shown in red), while any shared processor points (shown in white) are allowed to be optimized by Mesquite. After the optimization process, all shared processor points are averaged to obtain the final position. Experience shows that this averaging procedure is sufficient to maintain mesh quality during the course of the simulation process, while being computationally efficient.
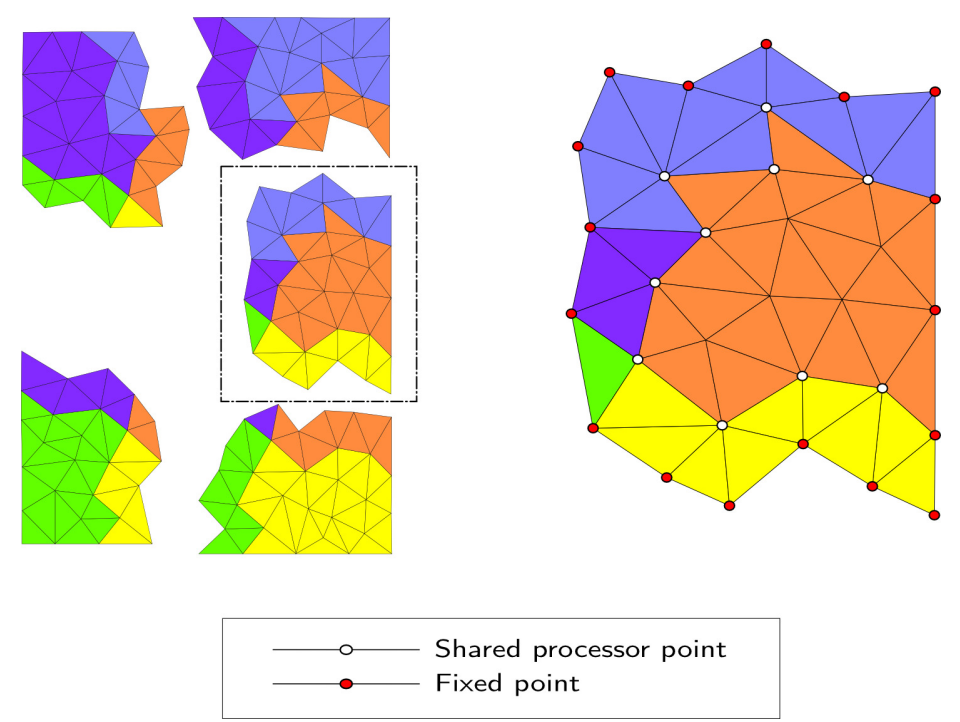

Figure 7: Shared processor points 


\subsection{Parallel Reconnection for Improved Mesh Quality}

The mesh adaptation procedure implemented in this work is a natural extension of the algorithms discussed previously, but with special consideration for edges and faces located on processor boundaries. For the parallel mesh adaptation procedure, all halo mesh entities are dealt with first, before the interior ones. If entities of the sub-domain were sent to a lower-ranked processor, then those entities must be left untouched, since the other processor may choose to modify the mesh topology locally.

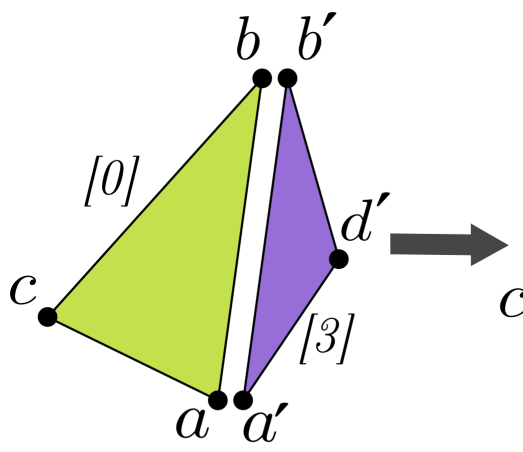

(a)

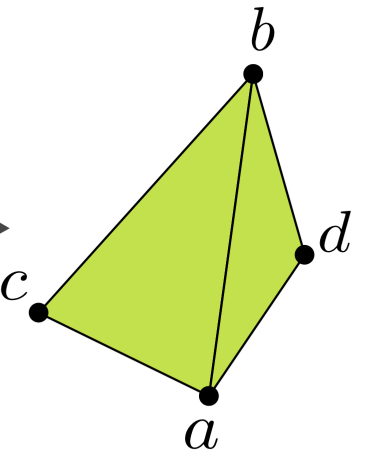

(b)

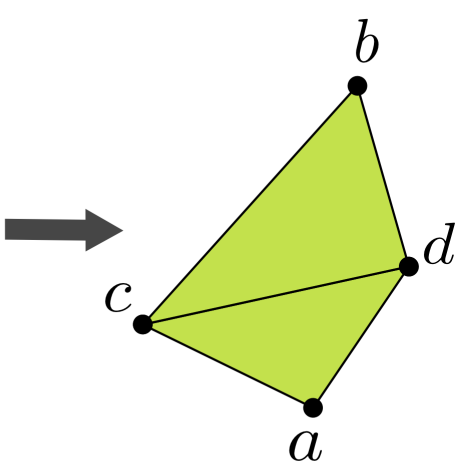

(c)

Figure 8: Cell migration for swapping in 2D

The concept of local cell-migration is now introduced to explain localized topology changes in this context. First, consider the two-dimensional case in Fig. 8(a), where triangle $a b c$ is located on processor 0 , while triangle $b^{\prime} a^{\prime} d^{\prime}$ is located on processor 3. At this point, since halo meshes have been transferred across neighboring processors, triangle $b^{\prime} a^{\prime} d^{\prime}$ is easily accessed by a look-up from the edge map (in this case, $\mathscr{X}_{e}^{3}$ ) for $a b$ to obtain $a^{\prime} b^{\prime}$ on the local halo mesh. The isolated point on triangle $b^{\prime} a^{\prime} d^{\prime}$ (namely, $d^{\prime}$ ) is now obtained, and the Delaunay test for edge $a b$ can be performed. If edge $a b$ fails the test, then triangle $b^{\prime} a^{\prime} d^{\prime}$ is migrated from the local halo mesh for processor 3 to processor 0 , as shown in Fig. 8(b). Since both triangles are located on processor $0, a b$ now becomes an interior edge, which is swapped conventionally. After this is done, the corresponding edge maps $\left(\mathscr{X}_{e}^{3}\right.$ and $\left.\mathscr{R}_{e}^{3}\right)$ are updated to include edges $a d$ and $b d$, which now represent the processor boundary. Since triangle $b^{\prime} a^{\prime} d^{\prime}$ is no longer present on processor 3 , an entry for cell-removal is made into a separate operations list. When multiple cells are migrated, the entries are made sequentially and accumulated until all operations on shared entities have been performed. This list is then transferred to the neighboring sub-domain (using MPI) for sequential removal. The operation-transfer process can be performed asynchronously to hide communication latency. Thus, while transfers occur in the background, topology changes for interior entities can be performed locally by each sub-domain. 
This approach to cell-migration makes local changes to inter-processor boundaries, and only involves cells that are present on the halo mesh. In contrast, the migration approach by Cavallo et al. [22] requires two global shifts to interprocessor boundaries, and a potentially large transfer of halo mesh connectivity and/or geometry information.

In $3 \mathrm{D}$, the cell quality optimization step is much more involved, since an edge can be shared by an arbitrary number of processors. An example is shown for edge $a b$ in Fig. 9(a), which is surrounded by 4 processors (the square-brackets denote processor rank). The functions that calculate hull quality [4] must now be modified to handle edges of this type. When the swapping algorithm encounters an edge on a processor boundary, the edge maps $\mathscr{X}_{e}^{j}$ are checked to obtain equivalent edges for each halo mesh $(j)$. All faces connected to edge $a b$ are collected, and for each triangular face $\left(f_{i}^{j}\right)$, the isolated vertex $v_{i}^{j}$ on the face (i.e., the point other than $a$ or $b$ ) is added to a list $\left(v_{p}\right)$. Since faces are duplicated on processor boundaries, only the isolated vertex corresponding to the lower sub-domain processor rank is added, thereby avoiding point duplicates (and therefore, justifying the requirement for halo meshes to possess boundary information).

A local coordinate system is now defined, with the origin passing through the edge center $\left(\mathbf{x}_{e}\right)$, and the z-axis along the vector $\mathbf{t}_{e}$ which is tangential to edge $a b$, as shown in Fig. 9(d). The choice of the $\mathrm{x}$-axis $\mathbf{d}_{x}$ depends on whether edge $a b$ lies on (i) a physical boundary (like a wall, for instance), or (ii) purely on a processor boundary (a pure processor edge, in this context, is one which has all attached faces either on processor boundaries or the interior of the mesh).

For the former case, the starting vertex $v_{s}=v_{i}^{j}$ is chosen such that it lies on a physical boundary patch. A pure processor edge is logically considered to be an interior edge and so, any choice from $\mathrm{v}_{p}$ would suffice for $v_{s}$. The axis $\mathbf{d}_{x}$ is then calculated by projecting the $\left(\mathrm{v}_{p}[i]-\mathbf{x}_{e}\right)$ vector on to the plane passing through $\mathbf{x}_{e}$, with $\mathbf{t}_{e}$ as its normal. The next step is to project all points in $\mathbf{v}_{p}$ on to the $\mathbf{d}_{x}-\mathbf{d}_{y}$ plane, and then compute the angles subtended by vertices with respect to $\mathbf{d}_{x}$ (given by $\theta$ in Fig. 9(d)). Now that all angles are obtained, the list is sorted by $\theta$ to obtain the counter-clockwise vertex ring $R$ around edge $a b$, which is then evaluated for minimum quality and optimized quality. If the optimized value is indeed better than the original hull-quality, then all cells surrounding $a b$ are migrated to the lowest-rank processor involved in the set (namely, processor 0 in Fig. 9(a)), and the corresponding maps are updated to account for the modified processor boundary. As with the $2 \mathrm{D}$ case, edge $a b$ now becomes internal to the mesh and can be removed using edge flipping. In situations where edge $a b$ lies on a physical boundary (considered to be an impure processor edge), the constructed vertex ring $R$ naturally starts and ends with boundary points, because all points are sorted by angles with respect to $\mathbf{d}_{x}$, which now aligns with $v_{s}$.

\subsection{Parallel Length Scale Resolution}

This section describes the approach taken to incorporate the length scale resolution operators, such as edge bisection and contraction, in a distributed- 


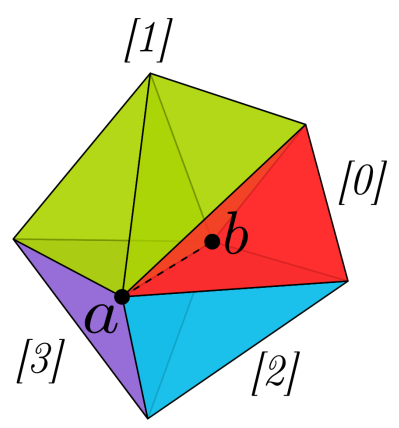

(a)

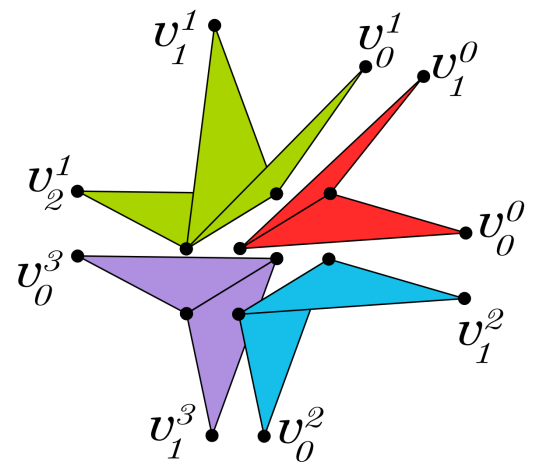

(c)

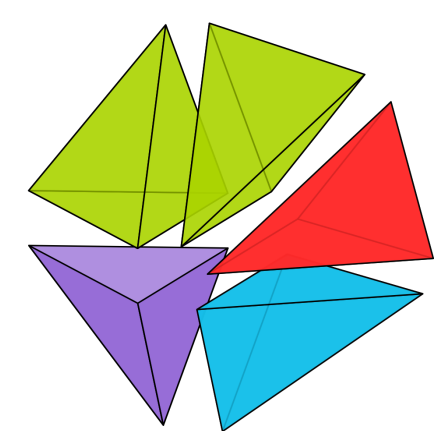

(b)

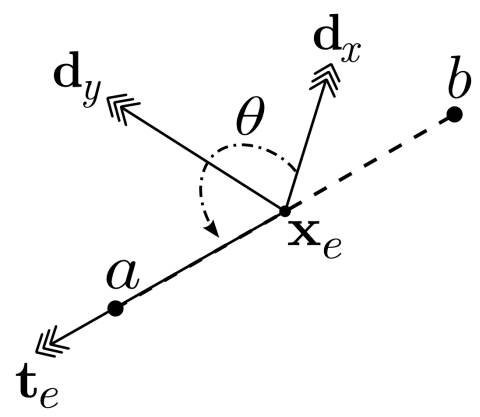

(d)

Figure 9: Parallel edge in 3D

memory parallel paradigm. Since these operators require a local length scale field to be defined throughout the mesh, the length scale algorithm defined in Section 2.4 must also be parallelized.

Conceptually, the algorithm is a face-cell wave that propagates the length scale value by a layer of cells at each iteration, using previously initialized values as input. Only a few major arrays in the algorithm need to be parallel-aware (and therefore updated) at each iteration. [38]

\subsection{Parallel Edge Refinement}

The next step is to incorporate coupled bisection and collapse operators that work across processor boundaries. The parallel bisection operation is relatively straightforward in $2 \mathrm{D}$ - for each parallel edge $a b$, the equivalent edge $a^{\prime} b^{\prime}$ is obtained using the edge map $\left(\mathscr{X}_{e}^{j}\right)$ on processor $j$. Since only one such coupling is possible, both edges are bisected to introduce points $c$ and $c^{\prime}$, respectively (shown in Fig. 10(a)). This procedure can be extended to 3D (shown in Fig. 10(b)), but there are now several entries for $a b$ in edge maps $\left(\mathscr{X}_{e}^{j} \ldots \mathscr{X}_{e}^{n}\right)$, since an arbitrary number of processors can share an edge. The first step is to accumulate and check all map edges $\left(a^{\prime} b^{\prime}\right)^{j} \ldots\left(a^{\prime} b^{\prime}\right)^{n}$ for bisection feasibility, 
followed by an actual bisection operation for all $\left(a^{\prime} b^{\prime}\right)^{j} \ldots\left(a^{\prime} b^{\prime}\right)^{n}$. Entries are then made into the operations list for each processor edge. Finally, the point maps $\left(\mathscr{X}_{p}^{j}, \mathscr{R}_{p}^{j}\right)$ are updated to include the new points introduced by bisection.
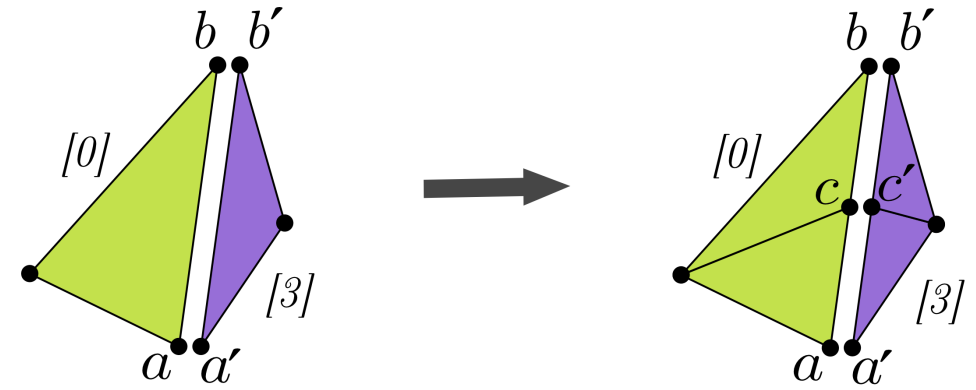

(a)
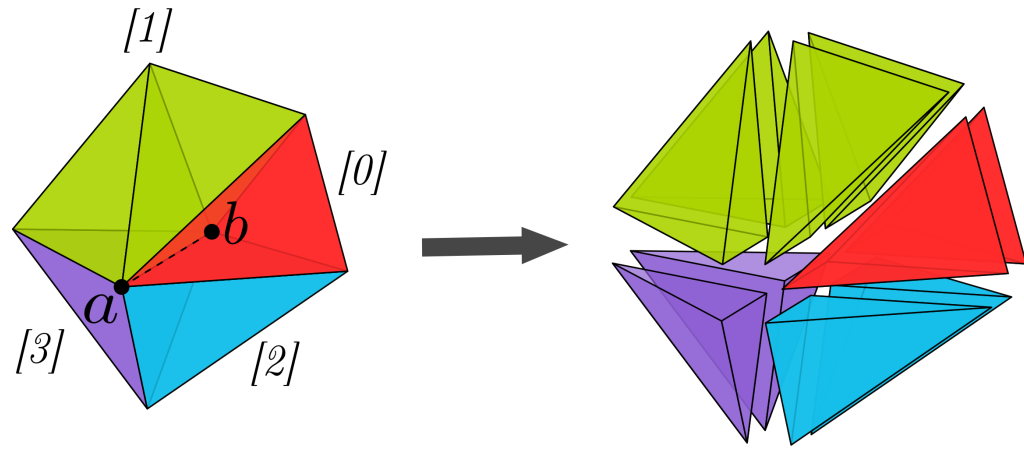

(b)

Figure 10: Parallel edge bisection in (a) 2D; and (b) 3D

Parallel edge collapse, on the other hand, tends to introduce a few additional complications. In two dimensions, the simplest case is one where an edge (and both its points) is shared by exactly two processors. In this situation, the operation is similar to the edge-bisection case - an initial check for feasibility is performed, followed by the actual collapse operation and updates to the operations list and point / edge maps. Occasionally, more complicated collapse situations can occur, such as the one depicted in Fig. 11. In this case, while edge $c d$ is shared by processors 0 and 3, point $c$ is additionally shared by processor 2. If a decision has been made by processor 0 to collapse edge $c d$, then an additional entry (to move point $\hat{c}$ ) has to be made in the operations list for processor 2 .

An additional scenario can occur in certain situations, where an operation can require the reassignment of a processor patch-face to another patch. In Fig. 12, for example, collapsing edge $c d$ requires edge $\dot{d} \dot{e}$ to be converted from a patch communicating with processor 3 , to a patch communicating with proces- 

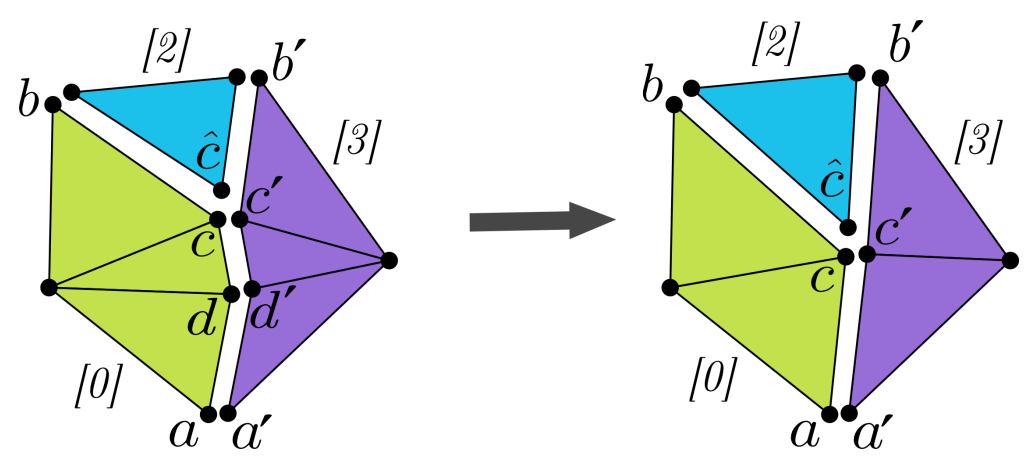

Figure 11: Parallel edge collapse in 2D

sor 2 (namely, edge $\hat{c} \hat{e}$ ). Also, if such a patch did not previously exist, then it must be created prior this step. While updating point maps, the entries for $\dot{d}$ is reassigned to $c$, since point $d$ was deleted during the collapse process. All these cases also carry over to the 3D situation, but with the additional possibility of multiply-connected processor edges.
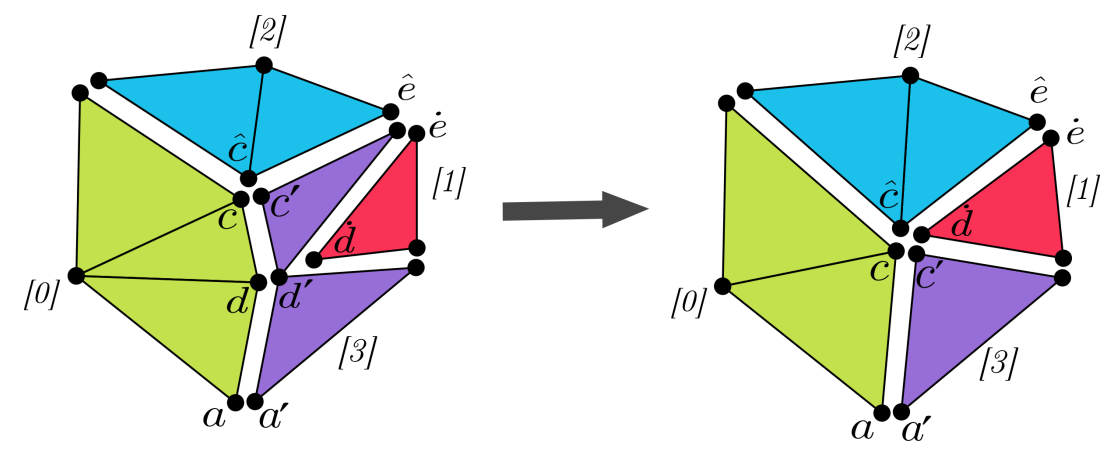

Figure 12: Patch conversion in 2D

In order to ensure that only one processor executes the required topological change, a priority system is applied. The processor identification numbers are initially listed in an array. The position in the array corresponds to the processor priority for executing topological changes. The array is rotated every time step, so that no one processor is at a disadvantage in implementing mesh adaptations. An example of the priorities amongst three processors would be:

TimeStep [0] : : Priority: (l $\left.\begin{array}{lll}0 & 1 & 2\end{array}\right)$

TimeStep [1] :: Priority: (1 20 0)

TimeStep [2] :: Priority: (2 01 ( 1$)$

TimeStep [3] : : Priority: (l $\left.\begin{array}{lll}0 & 1 & 2\end{array}\right)$

...

... 


\subsection{Parallel Field Remapping}

After parallel mesh reconnection, second-order accurate conservative field remapping using the methods described in Menon and Schmidt [49] tends to be complicated due to the fact that cells often migrate from one sub-domain to another. The task of finding intersections for elements adjacent to processor boundaries can sometimes become quite difficult, simply because all the required source-cells may not currently exist. This can be remedied, however, by recognizing that the halo meshes contain all the geometric information necessary for intersection calculations. If any of the source cells are found to be adjacent to processor boundaries, the halo meshes are also checked for intersections. The actual field variables needn't be present for this process and so, sub-sets of fields (corresponding to cells in halo meshes) can be transferred in the background using MPI while intersection calculations are being performed, thereby hiding communication latencies to a certain extent. Once all transfers have completed, the sub-domain fields are augmented with sub-fields corresponding to halo-meshes, and with some manipulation of the source-cell addressing, all fields can be remapped conventionally. Note that second-order conservative remapping requires cell-centered gradients, which must be transferred as well.

To conclude this section, the steps involved in the parallel reconnection algorithm are summarized. These steps are described from the stand-point of a particular sub-domain, since all sub-domains execute them in an identical sequence.

- Calculate a target length scale for all edges in the domain using the methods described in Section 3.4.

- Identify neighboring processors, including those that are connected only by points / edges.

- Construct halo meshes for each neighboring sub-domain. Send and receive halo meshes.

- Once halo meshes have been transferred, build maps for entities (point maps $\mathscr{X}_{p}$ and $\mathscr{R}_{p}$, edge maps $\mathscr{X}_{e}$ and $\mathscr{R}_{e}$, etc).

- Send and receive length scale values for halo cells.

- Coupled modifications:

- Initialize a stack with processor-coupled edges on halo meshes sent to other sub-domains, avoiding those sent to lower-ranked processors.

- Perform coupled-modifications using operations described in Section 3.3 and Section 3.5.

- Schedule transfer of the operations list to higher-ranked processors.

- Re-initialize stack with non-halo edges.

- Perform modifications using operations described in Section 3.3. 
- Synchronize coupled modifications:

- Wait for transfer of a mesh operations list from lower-ranked processors to complete.

- Sequentially execute operations from the list.

- Prepare and schedule transfer for sub-fields corresponding to halo meshes.

- Perform coupled intersection calculations using approach described in Section 3.6.

- Wait for sub-field transfers to complete, augment existing fields, and remap conventionally.

- Re-order boundaries so that processor-patches are matched both geometrically and topologically.

\section{Test Cases and Results}

The parallel adaptive meshing approach was tested using the OpenFOAM library, originally described in Weller et al. [50] and later in Jasak et al [51]. The OpenFOAM framework provides high-level $\mathrm{C}++$ interfaces that facilitate the development of CFD codes. The test cases were chosen in order to test the scalability and versatility of the algorithms. In particular, the tests offer a variety of prescribed and calculated boundary motion. The tests also vary whether internal moving boundaries, such as immersed objects, or external moving boundaries are present. A serial version of the dynamic remeshing algorithms has undergone flow accuracy validation studies in previous work [17] [18] [14] [19] [20] while being coupled to a moving mesh interface tracking method.

\subsection{Parallel Speed-up Tests}

An important consideration for the mesh-adaptation algorithm is to assess how well it scales with an increase in processor count. To investigate the scalability of the implementation, the first test case of a solid sphere (with a radius of 0.1 units) translating through a cubical domain (of side 1 unit) is used. The boundary motion is specified and an incompressible Navier Stokes solver calculates the surrounding fluid flow.

The initial domain is meshed with 1,562,629 tetrahedral cells, and subsequently split into sub-domains ranging between $4,8,16$ and 32 processors. The timings reported in this section were obtained using the QueenBee supercom-

puter from the Louisiana Optical Network Initiative (LONI), which is a part of the Teragrid project. The cluster consists of 668 compute nodes running RedHat Enterprise Linux, with each node containing dual Quad Core Xeon 64-bit processors operating at $2.33 \mathrm{GHz}$ with $8 \mathrm{~Gb}$ of RAM. All nodes are connected using a $10 \mathrm{~Gb} / \mathrm{sec}$ Infiniband network interface, using the MVAPICH-2 MPI implementation. 
Statistics for the simulations were collected over a period of 20 time-steps, where mesh-adaptation was used both with and without an incompressible flowsolver. Both timings are plotted against processor-count in Fig. 13. The mesh adaptation cost is observed to be approximately $60 \%$ of the total simulation time, of which about $80 \%$ is spent in the mesh smoothing process. Using the 4-processor case as a baseline, the speed-up for both adaptation and flow-solver is plotted in Fig. 14, and for adaptation alone in Fig. 15. Note that the ideal slope using 4-processors is now 0.25 , as opposed to the conventional slope of 1.0 . Interestingly, the 8- and 16-processor cases show a speed-up that is better than ideal, possibly owing to the large L2 cache sizes on the Xeon processor. The 32-processor case, however, shows the effect of increased communication costs, since each sub-domain now consists of approx. 48,000 cells, with a comparable size of processor boundaries.

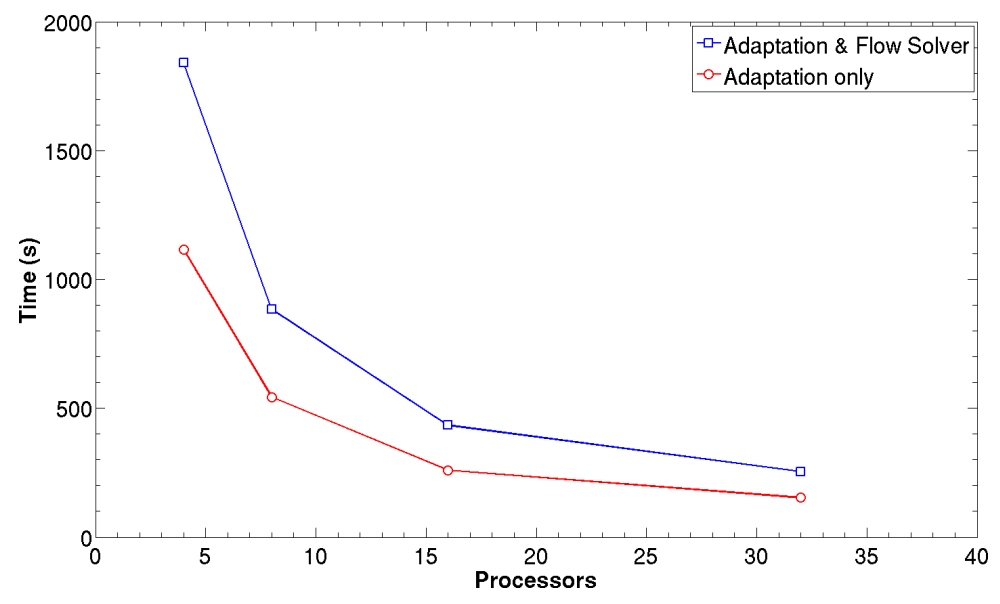

Figure 13: Solution time vs. processor count for 20 time-steps

\subsection{Internal Combustion Engine}

In the second case, to demonstrate the general applicability of the adaptation algorithm, the domain of an internal combustion engine designed for Gasoline Direct Injection (GDI) was selected. This is again a case of specified boundary motion, but here there are several different moving surfaces that represent outer boundaries. The motion is much more complex than the previous case, which only involved a single moving boundary. A further complication is that the piston moves into space that was previously occupied by the valves. This is known as an "interference engine" and is particularly difficult to model with structured meshes.

The domain consists of highly complicated piston and manifold geometries, with a pent-roof configuration and two intake valves, as shown in Fig. 16. The geometry is initially split into three parts, consisting of the two intake manifolds and the combustion chamber. 


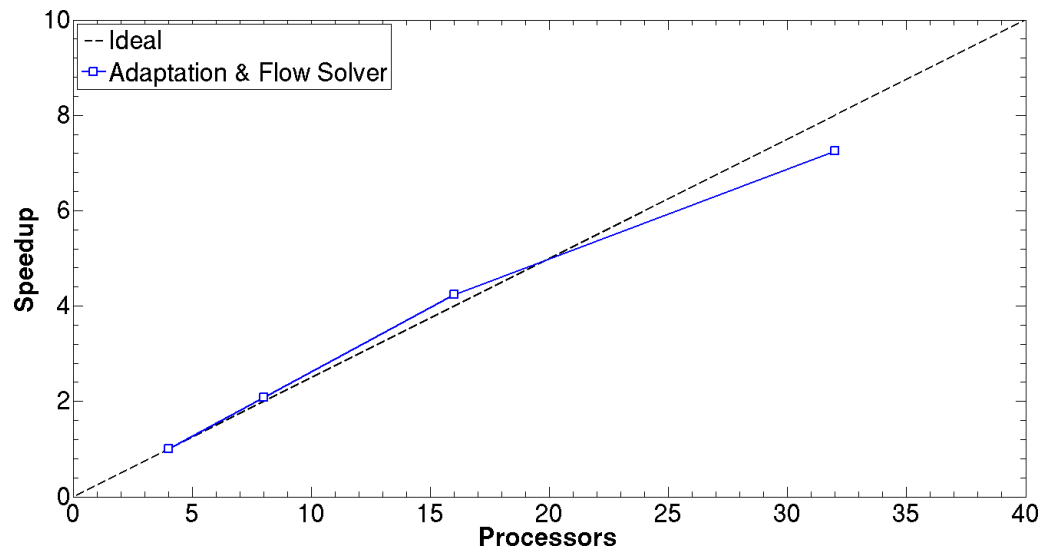

Figure 14: Speed-up for adaptation and flow-solver with a baseline of four processors

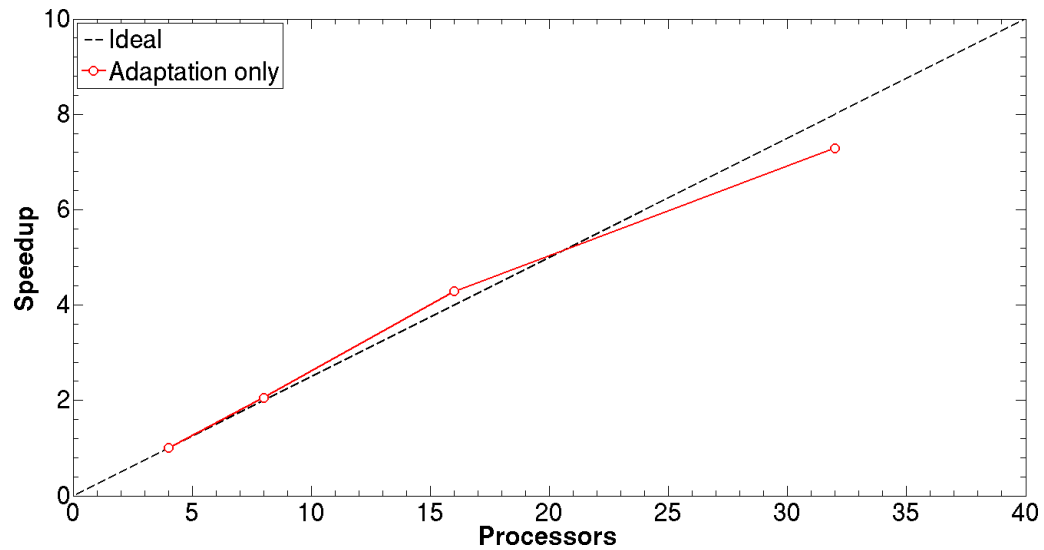

Figure 15: Speed-up for adaptation with a baseline of four processors

The engine bore is approximately $80 \mathrm{~mm}$, while the piston stroke is $45 \mathrm{~mm}$, and the valve lift is $4 \mathrm{~mm}$. At the start of the simulation, individual sub-domains have approximately 17,000 cells per processor, which increases to about 45,000 per processor at the mid-point, when the piston is at bottom-dead-center. The mesh is dynamically repartitioned at an arbitrarily chosen interval of 200 timesteps.

This case is also used to study mesh quality as the calculation evolves. Mesh quality is measured by a user-selected metric, which in the present case is an algebraic measure of mesh quality described by Knupp [52]. The quantity requires no expensive trigonmetric function evaluations, and is derived from the matrix that transforms an ideal tetrahedron into the particular cell of interest. It varies from zero to unity, and a typical initial mesh usually has a minimum quality of around 0.35 .

Figure 18 reveals that the mesh quality is maintined at an adequate value 

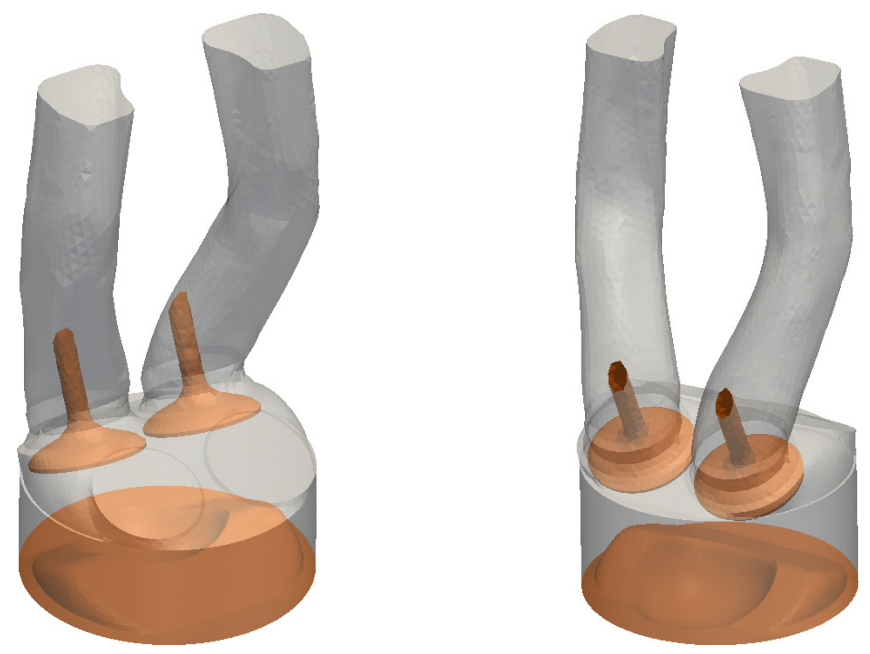

Figure 16: Geometry of the GDI internal combustion engine

during the computation. For the most part, the minimum cell quality is nearly constant. However, for discrete periods of time, lower quality cells can be created near concave boundaries, with all four cell vertices on the boundaries. Since all of the vertices lie on the boundary, interior mesh smoothing cannot improve the quality. Such cells persist briefly until an opportunity is found to remove them with topological operations, such as edge collapse. The user inputs include a minimum threshold that can be applied to the collapse operation to prevent degeneration below a certain quality value.

Fig. 19 shows the cell count history for each processor during the course of the simulation. The re-distribution steps can be clearly distinguished by the jumps in cell count as the simulation progresses. It is also interesting to note is that the histograms are not symmetric about the mid-point of the simulation, but stay roughly constant during the second half, with a rapid decrease in cell count towards the end. This is probably because the mesh smoother continuously maintains mesh quality to the point where edge contractions are deemed necessary to account for the reduced domain size at top-dead-center.

The total running time of the mesh adaptation on four processors was 5.7 hours, using a four core Intel Core2 Quad CPU running at $2.83 \mathrm{GHz}$ per core. Due to the absence of network hardware in this simulation, this is an unfair comparison, because it does not realistically exhibit communication latencies. An equivalent serial run, using identical simulation parameters, on one processor core took 11.45 hours. A cell-count history of the serial run is given in Fig. 20. The itemization of individual mesh adaptation costs is for the serial run is tabulated in Table 1.

A similar trend can be observed in the parallel run, as shown in Table 2 . The remapping step is slightly more time-consuming due to additional intersection checks against halo meshes. The re-ordering step also consumes a higher 


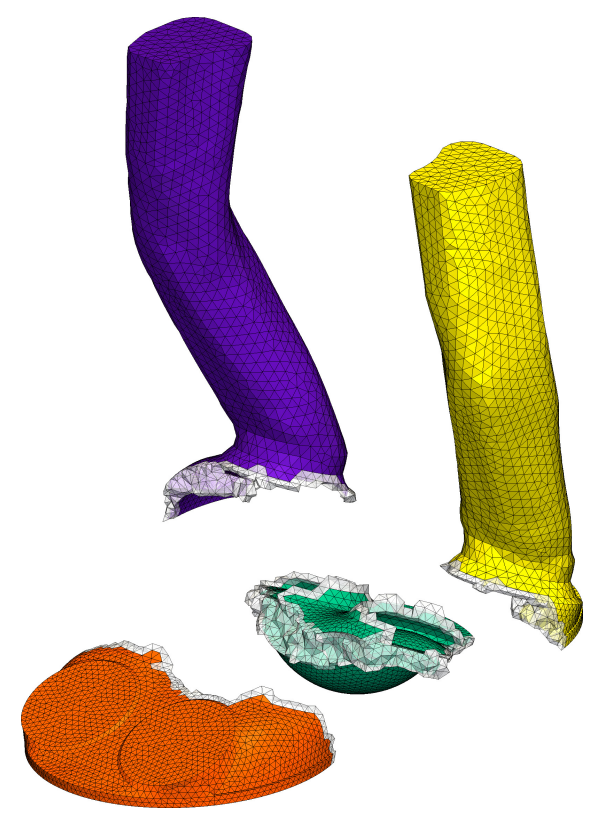

Figure 17: Parallel Decomposition of the GDI engine, showing halo meshes

\begin{tabular}{|l|r|r|}
\hline Operation & Time $(\mathrm{s})$ & Cost $(\%)$ \\
\hline Mesh smoothing & 34237.27 & $83.03 \%$ \\
Mesh reconnection & 5912.66 & $14.33 \%$ \\
Remapping & 30.55 & $0.07 \%$ \\
Mesh re-ordering & 1052.51 & $2.55 \%$ \\
\hline Total Adaptation Time & 41232.99 & $100.00 \%$ \\
\hline
\end{tabular}

Table 1: Itemization of mesh adaptation components for the serial run

percentage of the total cost, since processor patches have to be matched both geometrically and topologically after mesh reconnection, thereby requiring communication.

An additional assessment was performed in order to allow validation of the scheme. The ultimate measure of mesh quality is that the mesh supports highquality CFD results. The calculations were performed on a slightly different engine [53] where experimental data were available. The computationally predicted pressure was compared to experimental measurements, as shown in Figure 21. The experimental data are low-pass filtered to avoid oscillations that occur at valve seating and have nothing to do with the flow. The agreement between the computational and experimental results are excellent. 


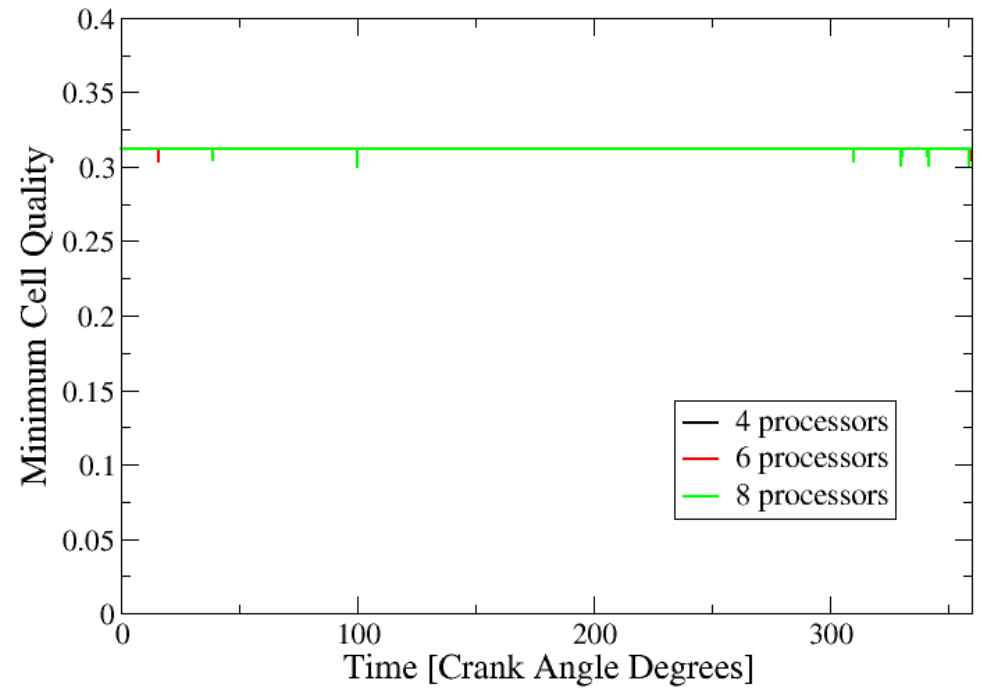

Figure 18: Minimum cell quality calculated as a function of time step during the computation for calculations with four, six, and eight processors.

\subsection{Six Degree-Of-Freedom Test}

In this third test case, an un-powered missile is allowed to move under the influence of gravity and aerodynamic forces. This is no longer a case of prescribed boundary motion. The sum of forces and torques, each in three dimensions, are used to calculate the motion and orientation of the missile. The surrounding gas is modeled as incompressible, laminar flow. The domain configurations are illustrated with the initial domain decomposition shown in Fig. 22. A close-up view of the missile and the calculated flowfield are shown in Fig. 23.

These results show the missile moving downwards under the effect of gravity, while being pushed leeward at the same time. Some rotation occurs during the

\begin{tabular}{|l|r|r|}
\hline Operation & Time $(\mathrm{s})$ & Cost (\%) \\
\hline Mesh smoothing & 16898.22 & $81.89 \%$ \\
Mesh reconnection & 2704.87 & $13.11 \%$ \\
Remapping & 205.34 & $0.99 \%$ \\
Mesh re-ordering & 824.58 & $4.01 \%$ \\
\hline Total Adaptation Time & 20633.01 & $100.00 \%$ \\
\hline
\end{tabular}

Table 2: Itemization of components for the parallel run 

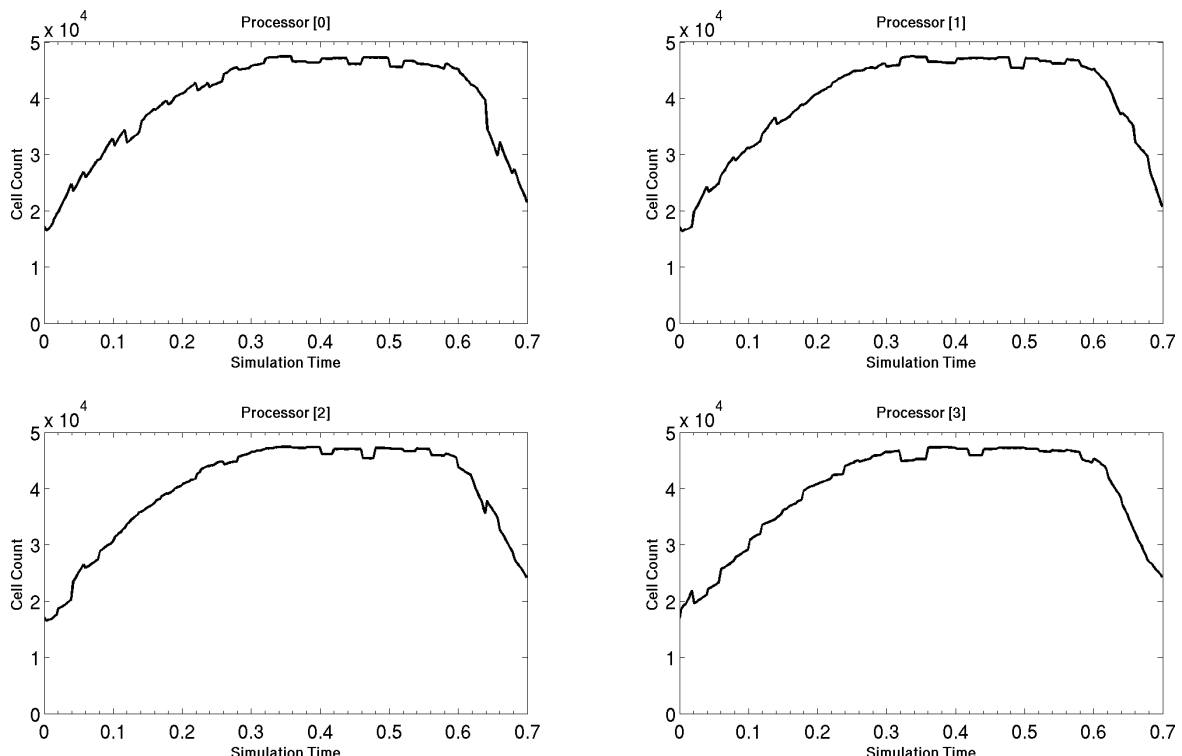

Figure 19: Cell count history for four processors

motion, as the nose of the missle drops. Note that the calculation resolves the separated flow occuring at the aft end of the missle.

\section{Conclusions}

The generality of the algorithms used in this work is demonstrated using several test cases involving meshes with arbitrary boundary motion. Deforming domain problems are vastly simplified using this framework, where only an initial mesh and a description of the moving boundaries are required, with no further user intervention needed. The mesh quality was found to remain good throughout a demonstration calculation. The extension of these algorithms to

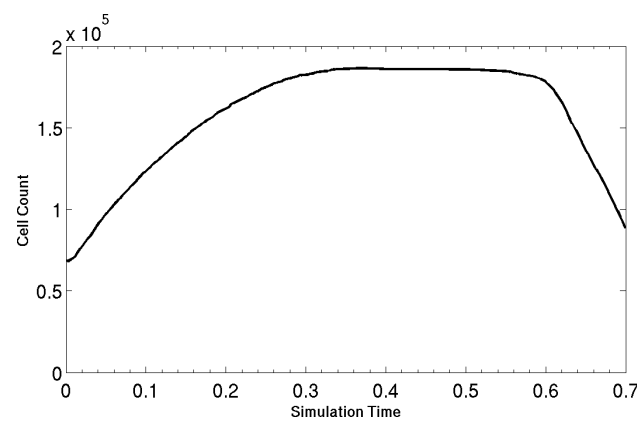

Figure 20: Cell count history for the serial run 


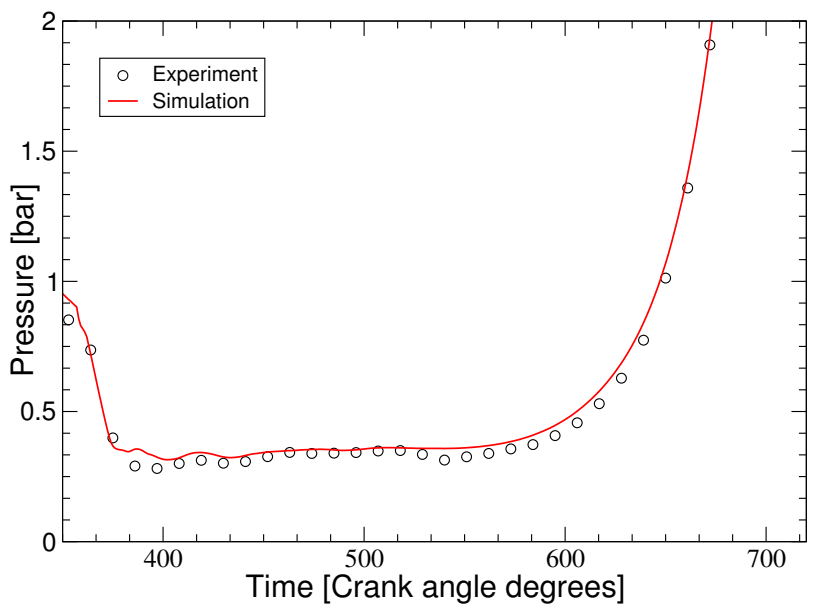

Figure 21: Measured and predicted pressure variation a gasoline direct injection engine. Time is measured in crank angle degrees after top dead center.

parallel paradigms considerably expands the ability of CFD methods to provide rapid solutions to problems with increased accuracy. While the cost of the scheme, especially mesh smoothing, is substantial, the adaptive mesh scheme demonstrated near-ideal speed-up.

While most of the cases considered in this work are transient in nature, these algorithms could be applied to facilitate other aspects of CFD, such as gridrefinement studies on steady-state calculations, and error-based mesh adaptation. All the work described in this article has been included in the OpenFOAM continuum mechanics library, which is free and open-source. It is anticipated that the algorithms devised in this work will be applied to a variety of situations involving dynamic meshes. The particular value is in complex boundary motion, where structured mesh approaches would be difficult. Examples of complex boundary motion might be boundaries that both move and deform, where the motion/deformation is not known prior to the calculation. 


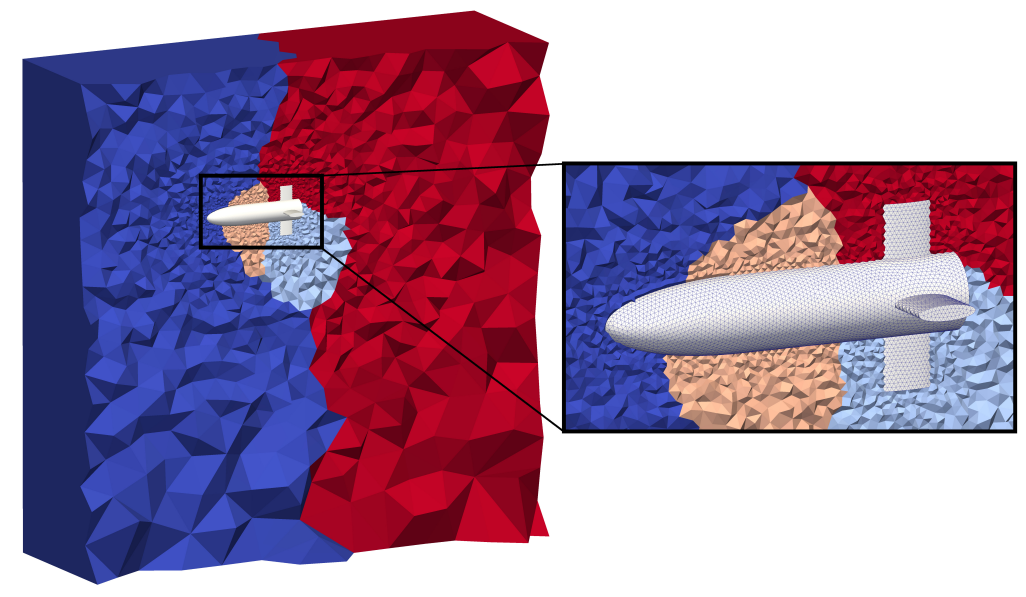

Figure 22: Processor decomposition of the free falling missile domain, colored by processor (4). Inset: An enlarged view to illustrate the missile hull surface mesh.

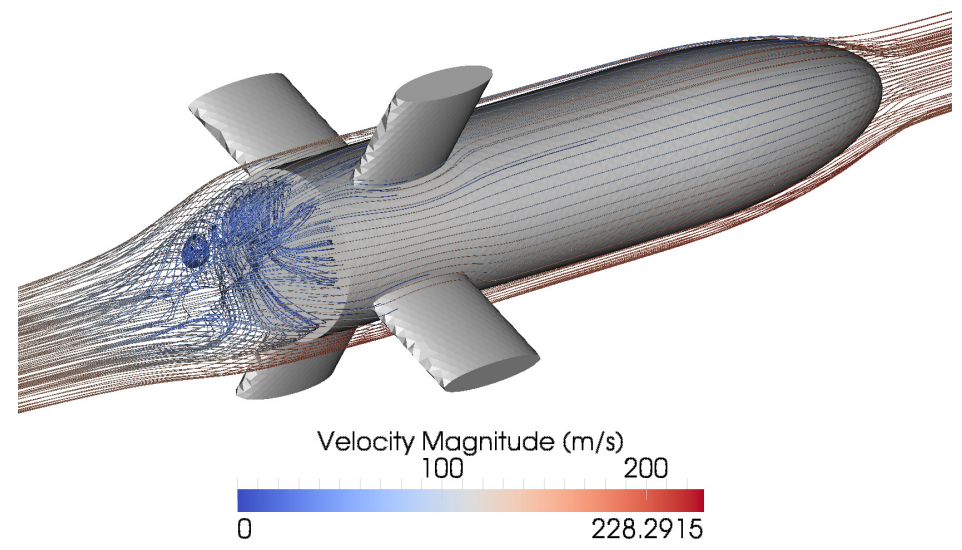

Figure 23: Streamlines around a missile moving under the influence of gravity and aerodynamic forces. 


\section{Acknowledgments}

We acknowledge the financial support of the Army Research Office under grant number W911NF-08-1-0171. Computations in this work utilized the Extreme Science and Engineering Discovery Environment (XSEDE), which is supported by National Science Foundation grant number OCI-1053575. We thank Dr. Chawki Habchi of IFP Energies Nouvelles for providing the Gasoline Direct Engine geometry and Dr. Federico Brusiani and Prof. Prof. Gian Marco Bianchi of the University of Bologna for the construction of the initial mesh.

\section{References}

[1] J. Benek, J. Steger, F. Dougherty, A flexible grid embedding technique with application to the Euler equations, AIAA 83-1944 .

[2] H. Tang, C. C. Jones, F. Sotiropoulos, An overset-grid method for 3D unsteady incompressible flows, Journal of Computational Physics 191 (2003) 567-600.

[3] S. Péron, C. Benoit, Automatic off-body overset adaptive Cartesian mesh method based on an octree approach, Journal of Computational Physics 232 (2013) 153-173.

[4] M. Dai, D. P. Schmidt, Adaptive Tetrahedral Meshing in Free-Surface Flow, J. Comput. Phys. 208 (1) (2005) 228-252.

[5] M. Wicke, D. Ritchie, B. M. Klingner, S. Burke, J. R. Shewchuk, J. F. O'Brien, Dynamic local remeshing for elastoplastic simulation, ACM Trans. Graph. 29 (2010) 49:1-49:11, ISSN 0730-0301, doi: http://doi.acm.org/10.1145/1778765.1778786.

[6] B. M. Klingner, J. R. Shewchuk, Aggressive Tetrahedral Mesh Improvement, in: M. L. Brewer, D. Marcum (Eds.), Proceedings of the 16th International Meshing Roundtable, Springer Berlin Heidelberg, ISBN 978-3540-75102-1, 3-23, 2008.

[7] W. Huang, R. Russell, Adaptive Moving Mesh Methods, Springer, 2010.

[8] C. Burstedde, O. Ghattas, G. Stadler, T. Tu, L. C. Wilcox, Parallel scalable adjoint-based adaptive solution of variable-viscosity Stokes flow problems, Computer Methods in Applied Mechanics and Engineering 198 (2126) (2009) 1691 - 1700, doi:10.1016/j.cma.2008.12.015.

[9] G. Gorman, Parallel anisotropic unstructured mesh optimisation and its applications, Ph.D. thesis, University of London, 2006.

[10] T. Baker, Mesh deformation and modification for time dependent problems, Int. J. Num. Meth. Fluids 43 (2003) 747-768. 
[11] G. Compére, E. Marchandise, J.-F. Remacle, Transient adaptivity applied to two-phase incompressible flows, Journal of Computational Physics 227 (2008) 1923-1942.

[12] T. Baker, Mesh movement and metamorphosis, Engineering with Computers 18 (2002) 188-198.

[13] P. Clausen, M. Wicke, J. R. Shewchuk, J. F. O'Brien, Simulating Liquids and Solid-liquid Interactions with Lagrangian Meshes, ACM Trans. Graph. 32 (2) (2013) 17:1-17:15, ISSN 0730-0301, doi:10.1145/2451236.2451243.

[14] K. Dieter-Kissling, M. Karbashi, H. Marschall, A. Javadi, R. Miller, D. Bothe, On the applicability of drop Profile Analysis Tensiometry at high flow rates using an interface tracking method, Colloids Surfaces A 441 (2014) 837-845.

[15] G. Stadler, M. Gurnis, C. Burstedde, L. C. Wilcox, L. Alisic, O. Ghattas, The Dynamics of Plate Tectonics and Mantle Flow: From Local to Global Scales, Science 329 (2010) 1033-1038.

[16] S. Quan, J. Lou, D. P. Schmidt, Modeling merging and breakup in the moving mesh interface tracking method for multiphase flow simulations, J. Comput. Phys. 228 (7) (2009) 2660 - 2675.

[17] K. Mooney, S. Menon, D. P. Schmidt, A Computational Study of Viscoelastic Droplet Collisions, in: ILASS-Americas 22nd Annual Conference on Liquid Atomization and Spray Systems, ILASS-Americas, 2010.

[18] K. Mooney, D. P. Schmidt, Finite Volume Simulations of the Collision of Viscoelastic Droplets using Adaptive Re-Meshing and Explicit Interface Tracking, in: ICLASS 2012 Triennial International Conference on Liquid Atomization and Spray Systems, International Conference on Liquid Atomization and Spray Systems, 2012.

[19] K. Dieter-Kissling, H. Marschall, A. Javadi, R. Miller, D. Bothe, Numerical method for coupled interfacial surfactant transport on dynamic surface meshes of general topology, Computers \& Fluids 109 (2015) 168-184.

[20] A. Batta, A. Class, K. Litfin, T. Wetzel, V. Moreau, L. Massidda, S. Thomas, D. Lakehal, D. Angeli, G. Losi, K. Mooney, K. V. Tichelen, Experimental and numerical investigation of liquid-metal free-surface flows in spallation targets, Nuclear Engineering and Design ISSN 0029-5493, doi: http://dx.doi.org/10.1016/j.nucengdes.2014.11.009.

[21] J. Qian, C. K. Law, Regimes of coalescence and separation in droplet collision, Journal of Fluid Mechanics 331 (1997) 59-80.

[22] P. Cavallo, N. Sinha, G. Feldman, Parallel unstructured mesh adaptation for transient moving body and aeropropulsive applications, in: Proceedings of the 42nd AIAA Aerospace Sciences Meeting and Exhibit, Reno, Nevada, 58 January, AIAA, 2004. 
[23] B. Erzincanli, M. Sahin, An arbitrary LagrangianEulerian formulation for solving moving boundary problems with large displacements and rotations, Journal of Computational Physics 255 (0) (2013) 660 - 679, ISSN 00219991, doi:http://dx.doi.org/10.1016/j.jcp.2013.08.038.

[24] F. M. Bos, Numerical simulation of flapping wing foil and wing aerodynamics, PhD Thesis, TU Delft .

[25] F. Brusiani, G. M. Bianchi, T. Lucchini, G. D'Errico, Implementation of a Finite-Element Based Mesh Motion Technique in an Open Source CFD Code, ASME Conference Proceedings 2009 (43406) (2009) 611-624.

[26] T. Lucchini, M. Fiocco, R. Torelli, G. D'Errico, Automatic Mech Generation for Full-Cycle CFD Modeling of IC Engines: Application to the TCC Test Case, in: SAE Congress, doi:10.4271/2014-01-1131, 2014.

[27] M. Brewer, L. Diachin, P. Knupp, T. Leurent, D. Melander, The Mesquite Mesh Quality Improvement Toolkit, in: 12th International Meshing Roundtable, Sandia National Laboratories report SAND 2003-3030P, Sept. 2003, 2003.

[28] F. J. Blom, Considerations on the Spring Analogy, Internat. J. Numer. Methods Fluids 32 (6) (2000) 647-668.

[29] W. Huang, R. D. Russell, Analysis of Moving Mesh Partial Differential Equations with Spatial Smoothing, SIAM J. Numer. Anal. 34 (3) (1997) 1106-1126, ISSN 0036-1429, doi: http://dx.doi.org/10.1137/S0036142993256441.

[30] S. Gosselin, C. Ollivier-Gooch, Tetrahedral mesh generation using Delaunay refinement with non-standard quality measures, International Journal for Numerical Methods in Engineering 87 (8) (2011) 795-820, ISSN 10970207 .

[31] L. A. Freitag, C. Ollivier-Gooch, Tetrahedral mesh improvement using swapping and smoothing, International Journal for Numerical Methods in Engineering 40 (1997) 3979-4002.

[32] E. Brière de L'Isle, P. L. George, Optimization of tetrahedral meshes, Institute for Mathematics and Its Applications 75 (1995) 97-127.

[33] G. Klincsek, Minimal triangulations of Polygonal domains, Ann. Discrete Math. 9 (1980) 121-123.

[34] H. Jasak, Error Analysis and Estimation for the Finite Volume Method with Applications to Fluid Flow, Ph.D. thesis, Imperial College, University of London, 1996.

[35] S. Muzaferija, Adaptive Finite Volume method for flow prediction using unstructured meshes and multigrid approach, Ph.D. thesis, Imperial College, University of London, 1994. 
[36] P. Coelho, J. C. F. Pereira, M. G. Carvalho, Calculation of laminar recirculating flows using a local non-staggered grid refinement system, Int. J. Num. Meth. Fluids 12 (6) (1991) 535-557.

[37] M. Dai, Numerical Simulation of Free Surface Flows using a Moving, Unstrucutred Mesh Method, Ph.D. thesis, University of Massachusetts, Amherst, 2005.

[38] S. Menon, A Numerical Study of Droplet Formation and Behavior Using Interface Tracking Methods, Ph.D. thesis, University of Massachusetts, 2011.

[39] W. Gropp, E. Lusk, N. Doss, A. Skjellum, A high-performance, portable implementation of the MPI message passing interface standard, Parallel Comput. 22 (6) (1996) $789-828$.

[40] G. Karypis, V. Kumar, A fast and high quality multilevel scheme for partitioning irregular graphs, SIAM J. Sci. Comput. 20 (1998) 359-392.

[41] K. Devine, E. Boman, L. Riesen, U. Catalyurek, C. Chevalier, Getting Started with Zoltan: A Short Tutorial, in: Proc. of 2009 Dagstuhl Seminar on Combinatorial Scientific Computing, 2009.

[42] F. Pellegrini, J. Roman, Scotch: A software package for static mapping by dual recursive bipartitioning of process and architecture graphs, in: H. Liddell, A. Colbrook, B. Hertzberger, P. Sloot (Eds.), High-Performance Computing and Networking, vol. 1067 of Lecture Notes in Computer Science, Springer Berlin / Heidelberg, 493-498, 1996.

[43] H. Jasak, Personal Communication, wikki Ltd., London UK, 13 March 2011.

[44] L. A. Freitag, M. Jones, P. Plassmann, A Parallel Algorithm for Mesh Smoothing, SIAM J. Sci. Comput. 20 (6) (1999) 2023-2040, doi:10.1137/S1064827597323208, URL http://link. aip.org/link/?SCE/20/2023/1.

[45] H. M. Tsai, J. Cai, F. Liu, A. S. F. Wong, Y. Zhu, Unsteady Flow Calculations with a Parallel Multiblock Moving Mesh Algorithm, AIAA Journal 39 (2001) 1021-1029.

[46] X. Jiao, P. J. Alexander, Parallel Feature-Preserving Mesh Smoothing, in: O. Gervasi, M. Gavrilova, V. Kumar, A. Lagana, H. Lee, Y. Mun, D. Taniar, C. Tan (Eds.), Computational Science and Its Applications ICCSA 2005, vol. 3483 of Lecture Notes in Computer Science, Springer Berlin / Heidelberg, 421-431, 2005.

[47] D. Guoy, T. Wilmarth, P. Alexander, X. Jiao, M. Campbell, E. Shaffer, R. Fiedler, W. Cochran, P. Suriyamongkol, Parallel Mesh Adaptation for Highly Evolving Geometries with Application to Solid Propellant Rockets, 
in: M. L. Brewer, D. Marcum (Eds.), Proceedings of the 16th International Meshing Roundtable, Springer Berlin Heidelberg, 515-534, 2008.

[48] M. R. Hestenes, E. L. Stiefel, Method of Conjugate Gradients for Solving Linear Systems, NBS J. Res. 29 (1952) 409-436.

[49] S. Menon, D. Schmidt, Supermesh construction for conservative interpolation on unstructured meshes: An extension to cell-centered finite-volume variables, Comput. Methods Appl. Mech. Engrg., 200 (2011) 2797-2804, doi:doi:10.1016/j.cma.2011.04.025.

[50] H. G. Weller, G. Tabor, H. Jasak, C. Fureby, A tensorial approach to computational continuum mechanics using object-oriented techniques, Comput. Phys. 12 (6) (1998) 620-631, ISSN 0894-1866, doi: http://dx.doi.org/10.1063/1.168744.

[51] H. Jasak, A. Jemcov, Z. Tuković, OpenFOAM: A C++ Library for Complex Physics Simulations, in: Z. Terze, C. Lacor (Eds.), Coupled Methods in Numerical Dynamics, Faculty of Mechanical Engineering and Naval Architecture, University of Zagreb, http://cmnd2007.fsb.hr, 2007.

[52] P. M. Knupp, Algebraic mesh quality metrics for unstructured initial meshes, Finite Elements in analysis and design 39 (3) (2003) 217-241.

[53] K. G. Stapf, S. Menon, D. P. Schmidt, M. Rieß, M. Sens, Charge Motion and Mixture Formation Analysis of a DISI Engine Based on an Adaptive Parallel Mesh Approach, in: SAE Congress, 2014. 